HERDER JAHRBUCH

HERDER YEARBOOK

1996 


\section{Beirat}

Martin Bollacher, Ruhr-Universität Bochum

Richard Critchfield, Texas A \& M University

Otto Dann, Universität Köln

Gonthier-Louis Fink, Université Strasbourg

Ulrich Gaier,Universität Konstanz

Hans Dietrich Irmscher, Universität Köln

Eva Knodt, Indiana University

Karl Menges, University of California, Davis

Michael Morton, Duke University

Kurt Mueller-Vollmer, Stanford University

Hugh Barr Nisbet, Sussex College Cambridge

Regine Otto, Stiftung Weimarer Klassik

Peter Hanns Reill, University of California, Los Angeles

Kurt Rudolph, Universität Marburg

Wilhelm Schmidt-Biggemann, Freie Universität Berlin

Helmut Schneider, Universität Bonn

Josef Simon, Universität Bonn

Jürgen Trabant, Freie Universität Berlin 


\title{
HERDER JAHRBUCH HERDER YEARBOOK 1996
}

\author{
Herausgegeben von \\ Wilfried Malsch, Hans Adler und Wulf Koepke
}

VERLAG J. B. METZLER

STUTTGART · WEIMAR 


\section{Dank:}

Die Edition dieses Bandes wurde zudem vom Vicechancellor for Research and Graduate Studies und vom Dean of Humanities and Fine Arts finanziell und vom Department of Germanic Languages and Literatures der University of Massachusetts in Amherst, Massachusetts, USA, in vielfältiger Weise und ebenso vom Metzler Verlag unterstützt.

Für Gutachten seien Martin Bollacher, Marcia Bunge, Richard Critchfield, Ulrich Gaier, Hans Dietrich Irmscher, Eva Knodt, Karl Menges, Kurt Mueller-Vollmer, Hugh Barr Nisbet, Regine Otto und Helmut Schneider bedankt.

Dank gebührt auch der Editionsassistentin Kristina von Held (Departement of Germanic Languages and Literatures, Amherst).

Die Deutsche Bibliothek - CIP-Einheitsaufnahme

Herder-Jabrbuch $=$ Herder yearbook. - Stuttgart ; Weimar : Metzler. Erscheint jährl. - Früher im Verl. Camden House, Columbia SC. Wurde früher nicht angezeigt. - Aufnahme nach 1997

NE: Herder yearbook

ISSN 0948-5279

ISBN 978-3-476-01466-5

ISBN 978-3-476-03672-8 (eBook)

DOI 10.1007/978-3-476-03672-8

Dieses Werk einschließlich aller seiner Teile ist urheberrechtlich geschützt.

Jede Verwertung außerhalb der engen Grenzen des Urheberrechtsgesetzes ist ohne Zustimmung des Verlages unzulässig und strafbar. Das gilt insbesondere für Vervielfältigungen,

Übersetzungen, Mikroverfilmungen und die Einspeicherung und Verarbeitung in elektronischen Systemen.

(C) 1997 Springer-Verlag GmbH Deutschland

Ursprünglich erschienen bei J. B. Metzlersche Verlagsbuchhandlung und Carl Ernst Poeschel Verlag GmbH in Stuttgart 1997 


\section{INHALT}

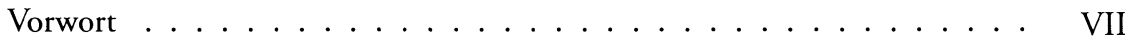

ARnd BOHM (Ottawa): Mixing Church and State.

Herder's Sermons on the Birth of Carl Friedrich . . . . . . . . . . . 1

Helmut Peitsch (Cardiff): French and German Masses in the Revolution.

Images and Concepts in Georg Forster's 1793 Writing on Paris and

Mainz ......................... 19

Christian Moser (Bonn): Der "Traum der schreibenden Person

von ihr selbst". Autobiographie und Subjektkonzeption bei

Johann Gottfried Herder . . . . . . . . . . . . . . . . . . . . 37

HEINRICH ClairmonT (Castrop Rauxel): ... un tableau vivant.

Herders physiologisch fundierte Psychologie, Lavaters Physiognomik und ein Disput in der Berliner Akademie der Wissenschaften . . . . . . . 57

ALAN CORKHILl (Brisbane): Herder and the Misuse of Language . . . . . . . 81

Robert S. Leventhal (Charlottesville): The Critique of the Concept.

Lessing, Herder, and the Semiology of Historical Semantics . . . . . . . . 93

DORIS KUHLES (Weimar): Herder-Bibliographie für 1993 und 1994 . . . . . 111

Namen- und Personenregister

Das Herder-Jahrbuch und die Internationale Herder-Gesellschaft . . . . . . 189

Adressen der Beiträger und Herausgeber . . . . . . . . . . . . . . . . . . 191 


\section{VORWORT}

Der vorliegende dritte Band des Herder-Jahrbuchs nimmt Anregungen auf, die das Herder-Jubiläum 1994 gebracht hat und führt die Auseinandersetzungen um das neue Herderbild der letzten zwei Jahrzehnte weiter. Außerdem erscheint in ihm erstmals eine Bibliographie der neuen Herder-Literatur, denn das Jahrbuch enthält von nun an in Kooperation mit der Stiftung Weimarer Klassik eine Fortsetzung der von Doris Kuhles besorgten, 1994 erschienenen Herder-Bibliographie.

Die Beiträge dieses Bandes behandeln historisch-politische, ideengeschichtliche, gattungstheoretische, linguistische und historisch-semantische Themen. So entwickelt Arnd Bohm aus der Herrscherkritik in Predigten von 1783 Herders religiös fundierten Begriff sozialer Gerechtigkeit als Grundmaß seines politischen Denkens und erweist damit die Unterstellung eines ssäkularisierten kulturellen Nationalismus` als nichtig. Wie Georg Forster in seinen Revolutionsschriften von 1793 die Probleme des in Masse und Elite geteilten Volks einem politisch aktivierbaren Gemeingeist aller konfrontiert, untersucht Helmut Peitsch vor dem Hintergrund der späteren kulturkritischen Debatte dieses Themas. Christian Mosers Analyse des Journals von 1769 zeigt im Licht neuer Theorien zu Autobiographie und Fiktion, wie die im Tagebuch intendierte Selbstanalye zur Produktionsstätte einer imaginären Ich-Identität wird. Heinrich Clairmont erläutert die verdeckte Auseinandersetzung Herders mit Lavaters Physiognomik im Kontext der Berliner Akademie-Diskussion über den sozialen Nutzen physiognomischer Urteile. Alan Corkhill eröffnet den Blick auf die wichtige, aber bisher noch nicht eigens untersuchte Sprachethik Herders und seine Betrachtungen über manipulativen und unabsichtlichen Sprachmißbrauch. Den kritischen `Dialog Herders mit Lessing über die Vorstellung: Wie die Alten den Tod gebildet, analysiert Robert Leventhal als Beispiel historisch-kulturellen Bedeutungswandels. Er führt dabei eine laufende Kontroverse über postmodernen 'Skeptizismus` und `kritischen Realismus` und über die Herder darin zugedachte aktuelle Bedeutung fort.

Das Manuskript dieses Bandes wurde im Mai 1996 druckfertig abgeschlossen. Für sein verspätetes Erscheinen, bedingt durch unvorhersehbare Probleme bei der $\mathrm{Zu}$ schußgewährung, wird um Nachsicht gebeten.

W. M. 